\title{
ANÁLISE DO DESEMPENHO DOS ALUNOS DE CURSOS SUPERIORES EM COMPUTAÇÃO NO ENADE - UMA ABORDAGEM USANDO MINERAÇÃO DE DADOS
}

\author{
Alexsander Figueiredo Silva, Raphael Magalhães Hoed e Pedro Fábio Saraiva \\ Instituto Federal de Educação, Ciência e Tecnologia do Norte de Minas Gerais - Campus Januária, Brasil
}

\begin{abstract}
RESUMO
A qualidade do ensino superior brasileiro gera impactos sociais em relação à formação de bons profissionais com a capacidade de promover o desenvolvimento do país. O Exame Nacional de Desempenho dos Estudantes (Enade) foi instituído com o propósito de analisar o conhecimento adquirido dos alunos de graduação brasileiros durante a sua formação acadêmica. Este artigo apresenta um estudo sobre o desempenho dos estudantes nos cursos superiores de computação com base nos microdados do Enade disponibilizados pelo Instituto Nacional de Estudos e Pesquisas Educacionais Anísio Teixeira (INEP). Foram utilizadas técnicas de mineração de regras de associação por meio do algoritmo Apriori, analisando-se quais fatores influenciam no desempenho dos estudantes ao realizar o exame em questão.
\end{abstract}

\section{PALAVRAS-CHAVE}

Apriori, Enade, Desempenho, Computação

\section{INTRODUÇÃO}

As avaliações de grande porte que são feitas no Brasil, desenvolvidas por órgãos públicos, têm um papel distinto e fundamental para a sociedade, pois visam obter informações sobre a eficiência e qualidade das organizações que proveem bens vitais para a população, tais como: saúde, educação e segurança. Esses dados são importantes, uma vez que, tendo em mão esses resultados, é possível avaliá-los para gerar ações interventivas com o objetivo de melhorar a qualidade na área com precariedade e aperfeiçoar a utilização dos recursos públicos.

Entre as diversas avaliações que são feitas no Brasil, encontra-se o Exame Nacional de Desempenho dos Estudantes (Enade). Este exame é composto por questões referentes a conteúdos gerais e específicos. As questões são feitas de forma incisiva, para que seja possível avaliar as habilidades acadêmicas e as competências profissionais adquiridas pelos estudantes durante seu respectivo curso de graduação, verificando-se também a qualidade de ensino das instituições de educação superior (IES). Além disso, o Enade busca reunir informações pessoais relacionadas às características socioeconômicas dos estudantes (Limana; Brito, 2005). O Enade não apenas avalia a performance de seus participantes, mas também permite determinar a qualidade dos cursos de graduação, sendo que o conhecimento dos fatores correlacionados ao bom desempenho é importante para que medidas mais eficazes sejam tomadas por autoridades políticas e dirigentes em universidades, no sentido de ampliar esse desempenho positivo.

Devido à representatividade que sistemas de avaliação como o Enade possuem, é importante que estudos sejam conduzidos para examinar a validade desses sistemas de avaliação e para verificar os fatores que influenciam o bom desempenho do aluno ao realizar o exame. O Enade possibilita avaliar a excelência dos cursos por intermédio do desempenho dos estudantes (Primi et al., 2006). Como diversos fatores, incluindo os institucionais, podem influenciar o desempenho dos alunos de graduação (Soares, 2004), torna-se importante compreender as causas que estão associadas ao bom desempenho discente e também aquelas associadas ao mau desempenho, visando, nesse último caso, mitiga-las. Dentre os fatores que podem contribuir para o desempenho dos discentes no Enade, podemos mencionar os relacionados a questões socioeconômicas, conforme será detalhado na Seção 2.1, tornando útil, nesse contexto, avaliar o questionário 
preenchido pelo estudante durante a realização deste exame, já que dispõe de questões deste tipo.

O objetivo geral dessa pesquisa é analisar os microdados do Enade disponibilizados pelo Instituto Nacional de Estudos e Pesquisas Educacionais Anísio Teixeira (INEP) para verificar quais fatores, obtidos por meio do preenchimento de formulário durante a realização da prova, estão relacionados ao bom e ao mau desempenho do aluno no Enade. Os microdados usados na pesquisa são os referentes ao ano de 2017, devido ao fato de serem os dados mais atuais do Enade que envolvem os cursos superiores da área de computação.

Este artigo está divido da seguinte forma: A Seção 2 mostra a fundamentação teórica, onde se encontra uma breve elucidação sobre o Enade, citando sua importância e representatividade e a técnica de mineração de dados utilizada; A Seção 3 mostra a metodologia empregada na pesquisa; A Seção 4 mostra os estudos realizados; A Seção 5 demonstra as conclusões obtidas ao longo do estudo.

\section{FUNDAMENTAÇÃO TEÓRICA}

Esta Seção é divida em duas subseções: a Subseção 2.1 discorre sobre o Enade, tratando de sua estrutura, a relevância de seu uso no Brasil e dos estudos relacionados ao tema e a Subseção 2.2 apresenta a técnica de mineração de dados de associação via algoritmo Apriori.

\subsection{Exame Nacional de Desempenho dos Estudantes}

O Enade é um componente curricular obrigatório nos cursos de graduação e tem sido utilizado não só para aferir a aprendizagem dos graduandos, mas também como instrumento de ranqueamento entre as IES. O discente não pode, portanto, se negar a fazer a prova do Enade. E pela relevância que ela apresenta para as IES, os professores, coordenadores de curso e gestores de maneira geral, não podem negligenciá-la, sob pena de impactar negativamente a avaliação da qualidade dos cursos.

O Enade compreende questões instrumentais relacionadas à formação do profissional e com o seu desenvolvimento como cidadão (Andriola, 2009). Este exame possui um questionário socioeconômico e cultural que deve ser preenchido por seus participantes e que, assim como a avaliação, também é obrigatório.

Conforme Rothen e Nasciutti (Rothen e Nasciutti, 2011) o Enade é estruturado da seguinte forma: a prova é aplicada para os alunos ingressantes e concluintes da instituição; os cursos são avaliados de forma trienal; a avaliação tem a mesma estrutura para todos os cursos: uma parte de formação geral e outra parte de conhecimentos específicos.

A Tabela 1 apresenta uma compilação de alguns autores que estudaram os dados do Enade e as respectivas técnicas empregadas no estudo:

Tabela 1. Estudos Envolvendo Dados do Enade

\begin{tabular}{|c|l|c|}
\hline Autor & \multicolumn{1}{|c|}{ O que estudou } & Técnicas utilizadas \\
\hline Caetano et al. (2015) & $\begin{array}{l}\text { Verificaram se existe diferença significativa entre as notas } \\
\text { dos alunos dos cursos de Ciências Contábeis das } \\
\text { modalidades de ensino a distância e presencial, resultantes } \\
\text { do Enade de 2009. }\end{array}$ & $\begin{array}{c}\text { Regressão simples e } \\
\text { múltipla; teste não } \\
\text { paramétrico de } \\
\text { Mann-Whitney }\end{array}$ \\
\hline $\begin{array}{c}\text { Nicolini, De Andrade e } \\
\text { Torres (2013) }\end{array}$ & $\begin{array}{l}\text { Compararam o desempenho acadêmico de universidades, } \\
\text { centros universitários e faculdades públicas e privadas, por } \\
\text { tipo de instituição de ensino superior e pelo número de } \\
\text { estudantes nelas matriculados, com base na performance } \\
\text { dos egressos no curso de Administração no Enade 2009. }\end{array}$ & Estatística descritiva. \\
\hline Ferreira (2015) & $\begin{array}{l}\text { Identificou as variáveis significativas na explicação do } \\
\text { resultado do Enade 2012 no curso de Ciências Contábeis. }\end{array}$ & $\begin{array}{c}\text { Estatística descritiva; } \\
\text { Modelo hierárquico linear. }\end{array}$ \\
\hline Gutterres (2015) & $\begin{array}{l}\text { Verificou a diferença de desempenho de estudantes } \\
\text { cotistas e não cotistas na participação da nota do Enade. }\end{array}$ & $\begin{array}{c}\text { Mínimos quadrados } \\
\text { ordinários; método de } \\
\text { decomposição de Oaxaca }\end{array}$ \\
\hline De Carvalho e Cerqueira & $\begin{array}{l}\text { Verificaram se, após cursar a graduação, o diferencial de } \\
\text { desempenho acadêmico continua inferior entre os cotistas } \\
\text { dos cursos de graduação em medicina e de direito, } \\
\text { comparado com aqueles que ingressaram por ampla } \\
\text { concorrência. }\end{array}$ & Estatística descritiva. \\
\hline
\end{tabular}




\begin{tabular}{|c|c|c|}
\hline $\begin{array}{c}\text { Da Paixão Rocha, Leles, e } \\
\text { Queiroz (2018) }\end{array}$ & $\begin{array}{l}\text { Verificaram a associação entre o desempenho de } \\
\text { estudantes concluintes de Nutrição no Enade e fatores } \\
\text { socioeconômicos, trajetória acadêmica e perfil da } \\
\text { instituição. }\end{array}$ & $\begin{array}{l}\text { Análise descritiva, } \\
\text { regressão linear simples e } \\
\text { regressão múltipla }\end{array}$ \\
\hline Brito (2015) & $\begin{array}{l}\text { Determinou como as características do corpo docente de } \\
\text { uma IES influenciam o desempenho dos concluintes de } \\
\text { seus cursos de graduação em Administração no Enade. }\end{array}$ & Regressão múltipla \\
\hline $\begin{array}{c}\text { Andrino Nogueira e Tsunoda } \\
(2015)\end{array}$ & $\begin{array}{l}\text { Analisaram a relação entre as características } \\
\text { socioeconômicas dos estudantes que frequentam o ensino } \\
\text { superior com o desempenho deles no Enade. }\end{array}$ & $\begin{array}{l}\text { Mineração de dados - } \\
\text { algoritmo C4.5 }\end{array}$ \\
\hline Leão et al. (2018) & $\begin{array}{l}\text { Utilizaram mineração de regras de associação (algoritmo } \\
\text { Apriori) para analisar os microdados do Enade nos anos de } \\
2014 \text { e } 2016 \text { nos cursos das instituições ligadas à } \\
\text { Universidade Luterana do Brasil (ULBRA), visando } \\
\text { identificar as reclamações mais relevantes dos alunos e a } \\
\text { partir delas propor melhorias no processo de ensino. }\end{array}$ & $\begin{array}{l}\text { Mineração de dados - } \\
\text { algoritmo Apriori }\end{array}$ \\
\hline Figueiró et al. (2018) & $\begin{array}{l}\text { Aplicaram a técnica clusterização aos microdados do } \\
\text { Enade do ano de 2014, formando grupos das IES do } \\
\text { Consórcio das Universidades Comunitárias Gaúchas } \\
\text { (COMUNG) que oferecem o curso de graduação em } \\
\text { Ciência da Computação }\end{array}$ & $\begin{array}{c}\text { Mineração de dados - } \\
\text { clusterização hierárquica }\end{array}$ \\
\hline $\begin{array}{l}\text { Vista, Figueiró e Chicon } \\
\text { (2017) }\end{array}$ & $\begin{array}{l}\text { Aplicaram a técnica clusterização aos microdados do } \\
\text { Enade do ano de } 2014 \text { para extrair informações referentes } \\
\text { ao desempenho dos acadêmicos do curso de Ciência da } \\
\text { Computação no Rio Grande do Sul - Brasil }\end{array}$ & $\begin{array}{l}\text { Mineração de dados - } \\
\text { clusterização hierárquica }\end{array}$ \\
\hline Landes e Manhães (2018) & $\begin{array}{l}\text { Analisaram o desempenho dos participantes do Enade na } \\
\text { área de computação nos anos de } 2005,2008,2011 \text { e } 2014 \text {. } \\
\text { Verificou-se predominância do sexo masculino entre os } \\
\text { concluintes que fizeram a prova, aumento gradativo das } \\
\text { idades dos alunos nos anos de } 2011 \text { e } 2014 \text { em } \\
\text { comparação com os anos de } 2008 \text { e } 2005 \text { e notas médias } \\
\text { nas provas de formação geral e conhecimentos específicos } \\
\text { abaixo de } 60 \% \text {. }\end{array}$ & Análise estatística \\
\hline
\end{tabular}

A Tabela 1 apresentada não esgota o tema referente ao estudo dos microdados do Enade. Diversos autores exploraram o assunto e foi possível perceber que os aspectos relacionados ao desempenho dos discentes no Enade são multifatoriais. Dentre os estudos relacionados à área de computação, todos são focados em um grupo de instituições de ensino específicas, exceto o estudo feito por Landes e Manhães, que utilizou dados de todo o país. A proposta deste estudo consiste também na utilização dos microdados referentes a todos os cursos de Computação do Brasil. Contudo, se diferencia da proposta dos autores mencionados quanto à abordagem, utilizando-se a mineração de regras de associação no presente estudo, e também quanto ao ano avaliado (ano de 2017). Os resultados obtidos com esse trabalho podem ser aplicados á realidade de diversas instituições de ensino, conforme será apresentado na Seção 5 (conclusões).

\subsection{Mineração de Regras de Associação}

O algoritmo Apriori será usado nesse trabalho para mineração das regras de associação. Procura-se descobrir associações importantes entre o bom ou o mau desempenho do aluno na prova do Enade e os dados presentes no questionário preenchido pelos estudantes.

De acordo com Romão et al. (Romão et al.,1999) "Uma das técnicas mais atraentes é a Mineração de Regras de Associação, que tem como destaque o algoritmo Apriori. Ele pode trabalhar com um número grande de atributos, gerando várias alternativas combinatórias entre eles."

De acordo com Hoed (Hoed, 2016), a mineração de regras de associação tem muitas aplicações comerciais em se tratando, por exemplo, de supermercados, quando se pode averiguar, a partir de um banco de dados, se a venda de um determinado produto também está associada à venda de outro produto. A descoberta de regras de associação desse tipo podem subsidiar decisões como melhor disposição das mercadorias no supermercado, colocando estrategicamente os produtos correlacionados uns próximos aos 
outros. "O objetivo, então, é encontrar todas as regras de associação relevantes entre os itens, do tipo $X($ antecedente $) \Rightarrow Y($ consequente)" (Romão et al.,1999). De acordo com Hoed (Hoed, 2016), a mineração de regras de associação não é útil apenas no contexto de transações comerciais, mas pode ser empregada também para análise de dados do ensino. No que diz respeito ao problema estudado neste artigo, pode-se verificar, por exemplo, se há associação entre o bom ou o mau desempenho dos alunos no Enade e a participação em projetos de pesquisa, atividades de extensão, monitorias, intercâmbios etc.

A descoberta de regras de associação pode ser decomposta em duas etapas, de acordo com Agrawal et al. (1993 apud Romão et al., 1999): localizar os conjuntos de itens (itemsets) que apresentam suporte superior ao mínimo definido à partida; utilizar os itemsets obtidos na etapa 1 para gerar as regras de associação do banco de dados. Algumas definições importantes sobre mineração de regras de associação: "A toda regra de associação A $\rightarrow$ B associamos um grau de confiança, denotado por conf (A $\rightarrow$ B)" (de Amo, 2004). O grau de confiança seria a probabilidade de que uma transação que tenha um item, também contenha o outro item. A Equação 1 a seguir, formaliza essa definição (de Amo, 2004):

$$
\operatorname{conf}(A \rightarrow B)=\text { número de transações que suportam }(A \cup B)
$$

número de transações que suportam $A$

De acordo com Ribeiro (Ribeiro, 2015), para encontrar regras consideradas fortes, além do suporte e da confiança, é também utilizada a medida lift, que é definida pela Equação 2:

$$
\begin{aligned}
\operatorname{lift}(A, B)= & \frac{P(A \cup B)}{P(A) P(B)}
\end{aligned}
$$

Ainda de acordo com Ribeiro (Ribeiro, 2015), "A ocorrência de um item A é independente de um item B se $\mathrm{P}(\mathrm{A} U \mathrm{U})=\mathrm{P}(\mathrm{A}) \mathrm{P}(\mathrm{B})$. Se não, existe uma correlação entre os itens”. Desta forma, se o valor da Equação 2 for menor que 1, então a ocorrência de A correlaciona-se negativamente com a ocorrência de B. Se o resultado for superior a 1, A e B se correlacionam positivamente, evidenciando que a ocorrência de A implica na ocorrência de B. Conforme será detalhado na Seção 3 (Metodologia), no âmbito desse estudo, só serão consideradas como válidas as regras obtidas cujo lift seja superior a 3 .

As fases de execução do algoritmo Apriori compreendem geração, poda, validação (de Amo, 2004). Resumidamente, sem entrar em detalhes sobre cada fase, na fase de geração são gerados os itemsets que tenham alguma chance de serem frequentes. na fase de poda são descartados os itemsets sem chances de serem frequentes e na última fase é calculado o suporte de cada um dos itemsets do conjunto (de Amo, 2004). O funcionamento do algoritmo Apriori é descrito da seguinte forma:

$\mathrm{Na}$ primeira passagem, o suporte para cada item individual (conjuntos-de-1-item) é contado e todos aqueles que satisfazem o suporte_mínimo são selecionados, constituindo-se os conjuntos-de-1-item frequentes (F1).

Na segunda iteração, conjuntos-de-2-itens candidatos são gerados pela junção dos conjuntos-de-1-item (a junção é feita através da função apriori-gen) e seus suportes são determinados pela pesquisa no banco de dados, sendo, assim, encontrados os conjuntos-de-2-itens frequentes. O algoritmo Apriori prossegue iterativamente, até que o conjunto-de-k-itens encontrado seja um conjunto vazio. (de Vasconcelos e de Carvalho, 2004).

\section{METODOLOGIA}

Neste trabalho foi utilizada a técnica de mineração de regras de associação usando o algoritmo Apriori e utilizou-se a metodologia de mineração de dados Cross Industry Standard Process for Data Mining (CRISP-DM) que compreende as seguintes etapas: compreensão do negócio, compreensão dos dados, preparação dos dados, modelação (aplicação das técnicas de mineração de dados), avaliação dos resultados e desenvolvimento.

$\mathrm{Na}$ etapa de compreensão do negócio foram feitos os estudos para a compreensão dos objetivos da pesquisa conforme descrito nas seções anteriores desse trabalho. 
$\mathrm{Na}$ etapa de compreensão dos dados foi feita a análise dos microdados do Enade disponibilizados no Portal do INEP (http://portal.inep.gov.br/microdados), sendo verificadas quais variáveis são importantes para o estudo com a utilização do software de planilha eletrônica Microsoft Excel. O Excel foi utilizado nessa etapa, pois a maioria dos dados analisados encontra-se em formato Comma Separated Values (CSV). Foram utilizados os microdados correspondentes ao último ano em que os cursos de computação foram avaliados (ano de 2017). As seguintes variáveis foram consideradas nesse estudo:

- NU_IDADE: Idade do Inscrito

- TP_SEXO: Tipo de sexo

- NT_GER: Nota Bruta da Prova

- NT_FG: Nota Bruta da Formação Geral

- NT_CE: Nota Bruta no Componente Específico

- Variáveis do Questionário Socioeconômico $(\mathrm{QE})$, que representam as variáveis do questionário dos estudantes, totalizando 81 variáveis.

Durante a preparação dos dados, foi feita a limpeza da base de dados removendo as variáveis que não são importantes para o estudo, utilizando nesse processo o software Excel. Algumas variáveis foram discretizadas para facilitar a análise dos dados: NT_GER, NT_FG, NT_CE e NU_IDADE.

Na discretização das variáveis de notas, foram utilizados os quartis matemáticos para classificar os valores. Em se tratando da variável NT_GER, notas entre 0 e 33,4 são consideradas notas baixas (abaixo do primeiro quartil), entre 33,4 e 42,9 notas abaixo da média (entre o primeiro e o segundo quartil), entre 42,9 e 53,1 notas médias (entre o segundo e o terceiro quartil) e acima de 53,1 notas altas (acima do terceiro quartil). Para a variável NT_FG, notas entre 0 e 39,4 são consideradas notas baixa (abaixo do primeiro quartil), entre 39,4 e 52,3 notas abaixo da média (entre o primeiro e o segundo quartil), entre 52,3 e 64 notas médias (entre o segundo e o terceiro quartil) e acima de 64 notas altas (acima do terceiro quartil). Para a variável NT_CE, notas entre 0 e 29,6 são consideradas notas baixas (abaixo do primeiro quartil), entre 29,6 e 40 notas abaixo da média (entre o primeiro e o segundo quartil), entre 40 e 51,4 notas médias (entre o segundo e o terceiro quartil) e acima de 51,4 notas altas (acima do terceiro quartil). Já a idade dos inscritos foi baseada na classificação usada pela United Nations Demographic Yearbook review (ONU, 2004). Inscritos com a faixa etária entre 15 e 24 anos são considerados Jovens, entre 25 e 59 anos são considerados Adultos e com 60 anos ou mais são considerados Idosos.

Durante a fase de modelação foi usado o algoritmo Apriori para verificar quais variáveis estão associadas ao bom ou mau desempenho nos cursos de computação, utilizando as notas de cada etapa da prova, sendo elas: NT_GER, NT_FG e NT_CE citadas na etapa anterior, relacionadas com o questionário respondido pelos participantes. Nesta etapa foi utilizado o software $\mathrm{R}$ versão 3.6.0. As regras foram filtradas considerando os seguintes parâmetros:

- Confiança mínima de $85 \%$.

- Suporte compreendido entre 0,01 e 0,05 .

- Lift igual ou superior a 3.

- Regras que não apresentam alguma das variáveis relacionadas à nota foram removidas do estudo, pois pretende-se localizar regras associadas ao rendimento do aluno.

No decorrer da fase de avaliação, os resultados gerados pelo algoritmo Apriori foram discutidos e analisados, o que será detalhado na Seção 4 deste artigo.

Durante a fase de desenvolvimento são discutidas ações no sentido de melhorar o desempenho dos alunos na prova, tendo em vista os resultados obtidos na fase anterior, conforme será detalhado na Seção 5.

\section{ESTUDOS REALIZADOS}

A Tabela 2 a seguir demonstra as saídas do software $\mathrm{R}$ ao aplicar o algoritmo Apriori. As regras apresentadas são apenas uma parcela das 75 que foram geradas pelo algoritmo. As 10 regras apresentadas compreendem aquelas com maior valor para o parâmetro lift dentre as 75 regras filtradas. Seguem os significados de cada uma das variáveis usadas: QE_I32 = "No curso você teve oportunidade de aprender a trabalhar em equipe."; QE_I41 = "A coordenação do curso esteve disponível para orientação acadêmica dos estudantes."; QE_I43 = "Foram oferecidas oportunidades para os estudantes participarem de programas, projetos ou atividades de extensão universitária."; QE_I50 = "O estágio supervisionado proporcionou experiências 
diversificadas para a sua formação.”; QE_I60 = "O curso disponibilizou monitores ou tutores para auxiliar os estudantes."; QE_I09 = "Qual alternativa a seguir melhor descreve sua situação financeira (incluindo bolsas)?”; QE_I19 = “Quem lhe deu maior incentivo para cursar a graduação?”; QE_I26 = "Qual a principal razão para você ter escolhido a sua instituição de educação superior?"; QE_I53 = "Foram oferecidas oportunidades para os estudantes realizarem intercâmbios e/ou estágios fora do país."; QE_I71 = "Você já tem experiência profissional no magistério, qual a forma de contrato? Assinale a alternativa mais relevante para você.".

Seguem os significados para as respostas atribuídas às variáveis:

- Variáveis QE_I32, QE_I41, QE_I43, QE_I50 e QE_I60: As respostas possíveis para essa questão variam em uma escala de um (1) a seis (6), onde 1 corresponde a "Discordo Totalmente" e 6 corresponde a "Concordo Totalmente". A resposta obtida nas regras geradas para essas variáveis, dentro da escala informada, foi CINCO.

- Variável QE_I53: A resposta UM corresponde a "Discordo Totalmente"

- Variável QE_I09: A resposta E corresponde a "Tenho renda e contribuo com o sustento da família".

- Variável QE_I19: A resposta A corresponde a "Ninguém".

- Variável QE_I26: A resposta A corresponde a "Gratuidade".

- Variáveis QE_I71: A resposta I corresponde a "Não tenho experiência no magistério".

Tabela 2. Regras de associação obtidas usando o Algoritmo Apriori

\begin{tabular}{|c|c|c|c|c|c|}
\hline $\begin{array}{l}\text { Num } \\
\text { Regra } \\
\end{array}$ & Item da Direita & Item da Esquerda & Suporte & Confiança & Lift \\
\hline 1 & $\{$ NT_CE=NOTA_ALTA,QE_I60=CINCO $\}$ & $\Rightarrow\{$ NT_GER=NOTA_ALTA $\}$ & 0,04 & 0,88 & 3,53 \\
\hline 2 & $\left\{\mathrm{NT} \_\mathrm{CE}=\mathrm{NOTA}\right.$-ALTA,QE_I32=CINCO $\}$ & $\Rightarrow\{$ NT_GER=NOTA_ALTA $\}$ & 0,04 & 0,88 & 3,52 \\
\hline 3 & $\{$ NT_CE=NOTA_ALTA,QE_I50=CINCO $\}$ & $\Rightarrow\{$ NT_GER=NOTA_ALTA $\}$ & 0,04 & 0,88 & 3,52 \\
\hline 4 & $\{$ NT_CE=NOTA_ALTA,QE_I43=CINCO $\}$ & $\Rightarrow\{$ NT_GER=NOTA_ALTA $\}$ & 0,04 & 0,87 & 3,51 \\
\hline 5 & $\left\{\mathrm{NT} \_\mathrm{CE}=\mathrm{NOTA}=\mathrm{ALTA}, \mathrm{QE} \_\mathrm{I} 41=\mathrm{CINCO}\right\}$ & $\Rightarrow\{$ NT_GER=NOTA_ALTA $\}$ & 0,04 & 0,87 & 3,51 \\
\hline 6 & $\{$ NT_CE=NOTA_BAIXA,QE_I71=I $\}$ & $\begin{array}{l}\Rightarrow\{\text { NT_GER= } \\
\text { NOTA_BAIXA }\}\end{array}$ & 0,04 & 0,88 & 3,54 \\
\hline 7 & $\{$ NT_CE=NOTA_BAIXA,QE_I09=E $\}$ & $\begin{array}{l}\Rightarrow>\{\text { NT_GER= } \\
\text { NOTA_BAIXA }\}\end{array}$ & 0,04 & 0,85 & 3,42 \\
\hline 8 & $\{$ NT_CE=NOTA_BAIXA,QE_I19=A $\}$ & $\begin{array}{l}\Rightarrow \text { PNT_GER }= \\
\text { NOTA_BAIXA }\}\end{array}$ & 0,03 & 0,85 & 3,40 \\
\hline 9 & $\{$ NT_GER=NOTA_BAIXA,QE_I26=A $\}$ & $\Rightarrow\{$ NT_CE $=$ NOTA_BAIXA $\}$ & 0,03 & 0,85 & 3,37 \\
\hline 10 & $\{$ NT_GER=NOTA_BAIXA,QE_I53=UM $\}$ & $\Rightarrow\{$ NT_CE $=$ NOTA_BAIXA $\}$ & 0,03 & 0,85 & 3,36 \\
\hline
\end{tabular}

Para melhor compreensão, seguem os comentários das regras encontradas: Regra 1 - $88 \%$ dos estudantes que apresentaram nota alta em NT_CE e concordam que o curso disponibilizou monitores ou tutores para auxiliar os estudantes, também apresentaram nota alta em NT_GER; Regra $2-88 \%$ dos estudantes que apresentaram nota alta em NT_CE e tiveram oportunidade de aprender e trabalhar em equipe, também apresentaram nota alta em NT_GER; Regra 3 - 88\% dos estudantes que apresentaram nota alta em NT_CE e afirmam que o estágio supervisionado proporcionou experiências diversificadas para a sua formação, também apresentaram nota alta em NT_GER; Regra 4 - 87\% dos estudantes que apresentaram nota alta em NT_CE e afirmam que foram oferecidas oportunidades para os estudantes participarem de programas, projetos ou atividades de extensão universitária, também apresentaram nota alta em NT_GER; Regra 5 - 87\% dos estudantes que apresentaram nota alta em NT_CE e afirmam que a coordenação do curso esteve disponível para orientação acadêmica dos estudantes, também apresentaram nota alta em NT_GER; Regra 6 - 88\% dos estudantes que apresentaram nota baixa em NT_CE e não tiveram experiência profissional no magistério, também apresentaram nota baixa em NT_GER; Regra $7-85 \%$ dos estudantes que apresentaram nota baixa em NT_CE e que têm renda e contribuem com o sustento da família, também apresentaram nota baixa em NT_GER; Regra 8-85\% dos estudantes que apresentaram nota baixa em NT_CE e não tiveram incentivo da parte de alguém para cursar graduação, também apresentaram nota baixa em NT_GER; Regra 9 - 85\% dos estudantes que apresentaram nota baixa em NT_GER e escolheram a instituição de educação superior por ser 
gratuita, também apresentaram nota baixa em NT_CE; Regra $10-85 \%$ dos estudantes que apresentaram nota baixa em NT_GER e que discordam que foram oferecidas oportunidades para os estudantes realizarem intercâmbios e/ou estágios fora do país, também apresentaram nota baixa em NT_CE.

\section{CONCLUSÕES}

O referente trabalho mostrou, utilizando técnicas de mineração de regras de associação, fatores que se relacionam diretamente com o desempenho dos participantes do Enade em cursos superiores de Computação. Encontraram-se evidências, em relação aos alunos com mau desempenho, de que esses participantes, em maioria: não tem apoio externo para ingressarem na graduação; possuem renda, porém essa renda é destinada para o sustento da família; não tem experiência profissional no magistério; escolhem a instituição de ensino baseada no seu custo, ou seja, a preferência de instituições gratuitas está à frente de escolhas em relação à reputação de qualidade da instituição, demonstrando que o fator econômico reflete diretamente nessa categoria para escolha de instituição; faltam oportunidades para que os estudantes realizarem intercâmbios ou estágios fora do país, sendo este um fator que agregaria experiências acadêmicas muito positivas ao processo de formação do estudante.

Em relação ao bom desempenho, as regras demonstram que as instituições onde estes alunos estudam, oferecem um maior apoio no decorrer da graduação, como o fato do curso disponibilizar monitores ou tutores para auxílio dos estudantes, de supervisionar os alunos no estágio, proporcionando experiências diversificadas com oportunidades de aprender e trabalhar em equipe, agregando a experiência social, com a coordenação sempre disponível para a orientação acadêmica. Os fatos citados demonstram que uma instituição com uma boa estruturação e boas políticas de acompanhamento do discente que vão além da aula convencional, proporcionam aos estudantes um melhor desempenho no Enade.

Já entre os participantes que apresentaram mau desempenho, percebemos a vinculação de fatores sócio-culturais e econômicos como: a falta de incentivo, seja ele familiar ou governamental, e a escolha de instituições gratuitas, em vez de se ponderar, como principal fator de escolha, a qualidade dessas instituições.

Os fatores que podem influenciar no desempenho de um aluno são bem mais complexos do que as regras encontradas nessa pesquisa, visto que, o desempenho depende não só do ambiente em que o aluno realizou sua graduação, como também fatores socioeconômicos, pessoais entre outros tipos que requerem estudos mais aprofundados. No entanto, analisando as regras exploradas, é possível perceber que um dos principais fatores, remete a problemas institucionais que podem ser melhorados e tratados com melhor atenção por parte dos dirigentes educacionais. É necessário buscar estratégias, sejam elas governamentais ou vindas de dentro da própria instituição de ensino, que consigam melhorar o ambiente universitário para os estudantes, propiciando a formação de profissionais mais qualificados, inclusive para atuar no magistério. Espera-se que esse trabalho possa servir como subsídio para pesquisas mais aprofundadas nessa área e que possa contribuir com a comunidade acadêmica no sentido de mitigar os problemas existentes na educação superior brasileira.

\section{AGRADECIMENTO}

Ao Instituto Federal de Educação, Ciência e Tecnologia do Norte de Minas Gerais (IFNMG) - Campus Januária pelo apoio concedido por meio da disponibilização de infraestrutura (laboratório e computadores) para realização dessa pesquisa, tal como a concessão de bolsa de iniciação científica.

\section{REFERÊNCIAS}

Andrino Nogueira, E. D.; Tsunoda, D. F., 2015. Mineração de dados para análise da relação entre as características socioeconômicas de concluintes do ensino superior e o desempenho desses estudantes no enade 2012. Revista Percurso, Vol. 15, No. 1, pp 23.

Andriola, W. B., 2009. Fatores institucionais associados aos Resultados do Exame Nacional de Desempenho Estudantil (Enade): estudo dos cursos de graduação da Universidade Federal do Ceará (UFC). Revista Electrónica Iberoamericana sobre Calidad, Eficacia y Cambio en Educación (REICE). Madrid, Espanha. 
Brito, T. F., 2015. Corpo docente: fatores determinantes do desempenho discente no ENADE. Catálogo da Universidade de São Paulo (USP). São Paulo, Brasil.

Caetano, C. C. R.; Cardoso, T. A. O.; Miranda, G. J.; De Freitas, S.C., 2015. Desempenho no Enade em Ciências Contábeis: Ensino a Distância (EAD) versus presencial. Revista Universo Contábil. Blumenau, Santa Catarina, Brasil.

Da Paixão Rocha, A. L.; Leles, C. R.; Queiroz, M. G., 2018. Fatores associados ao desempenho acadêmico de estudantes de Nutrição no Enade. Revista Brasileira de Estudos Pedagógicos. Brasília, Distrito Federal, Brasil.

De Carvalho, M. M.; Cerqueira, G. S., 2015. Análise do Desempenho Acadêmico dos Cotistas dos Cursos de Medicina e Direito no Brasil. Grupo Estratégico de Análise da Educação Superior no Brasil (GEA). Rio de Janeiro, Brasil.

De Vasconcelos, L. M. R.; De Carvalho, C. L., 2004. Aplicação de Regras de Associação para Mineração de Dados na Web. Instituto de Informática da Universidade Federal de Goiás. Goiás, Brasil.

De Amo, S., 2004. Técnicas de Mineração de Dados. Dissertação, Universidade Federal de Uberlândia. Minas Gerais, Brasil.

Ferreira, M. A., 2015. Determinantes do desempenho discente no Enade em cursos de Ciência Contábeis. Dissertação, Universidade Federal de Uberlândia (UFU). Minas Gerais, Brasil.

Figueiró, M. F.; Vista, N. P. B.; Barasuol, J. B.; Chicon, P. M. M.; Ansuj, A. P, 2018. Análise de Agrupamento Hierárquico aplicada aos microdados do ENADE do curso de graduação em Ciência da Computação. Revista Eletrônica Argentina-Brasil de Tecnologias da Informação e da Comunicação. Rio Grande do Sul, Brasil.

Gutterres, R. S., 2015. Alunos que ingressaram no ensino superior por ações afirmativas apresentam melhor desempenho? Uma análise empregando a decomposição de Oaxaca para o ENADE 2012. Dissertação, Pontifícia Universidade Católica do Rio Grande do Sul (PUCRS). Rio Grande do Sul, Brasil.

Hoed, R. M., 2016. Análise da evasão em cursos superiores: o caso da evasão em cursos superiores da área de Computação. Dissertação de Mestrado, Universidade de Brasília (UnB). Brasília, Distrito Federal, Brasil.

Landes, F. B.; Manhães, L. M. B., 2018. Análise dos exames do enade para os cursos de computação utilizando o software R. Anais do Seminário Internacional de Estatística com R. Rio de Janeiro, Brasil.

Leão H. A. T.; Canedo E. D.; Ladeira M.; Fagundes F., 2018. Mining ENADE Data from the Ulbra Network Institution. Information Technology - New Generations. Advances in Intelligent Systems and Computing, vol 738. Springer, Cham.

Limana, A.; Brito, M. R. F., 2005. O Modelo de Avaliação Dinâmica e o Desenvolvimento de Competências: algumas considerações a respeito do Enade. Rede de Avaliação Institucional da Educação Superior (RAIES), da Universidade Estadual de Campinas (UNICAMP). Campinas, São Paulo, Brasil, pp. 12-14.

Nicolini, A. M.; De Andrade, R. O. B.; Torres, A. A. G., 2013. Comparando os Resultados do Enade 2009 por número de instituições e número de estudantes: como ainda o desempenho acadêmico dos cursos de administração? Administração: Ensino e Pesquisa (RAEP). Rio de Janeiro, Brasil.

ONU, 2004. United Nations Demographic Yearbook review. United Nations, Department of Economic and Social Affairs Statistics Division, Demographic and Social Statistics Branch. Estados Unidos, pp. 1-2.

Primi, R.; De Carvalho, L. F.; Miguel, F. K.; Da Silva, M. C. R., 2006. Análise do funcionamento diferencial dos itens do Exame Nacional do Estudante (Enade) de psicologia de 2006. Psico-UFS. São Paulo, Brasil, pp. 380-382.

Ribeiro, A. C., 2015. Correlação e visualização de alertas de segurança em redes de computadores. Dissertação de Mestrado, Universidade Estadual Paulista - Campus de São José do Rio Preto. São José do Rio Preto, São Paulo, Brasil.

Romão, W.; Niederauer, C. A.; Martins, A.; Tcholakian, A.; Pacheco, R. C.; Barcia, R. M., 1999. Extração de regras de associação em C\&T: O algoritmo Apriori. XIX Encontro Nacional em Engenharia de Produção. Florianópolis, Santa Catarina, Brasil, pp. 34, 37-39.

Rothen, J. C.; Nasciutti, F., 2011. A educação superior em prova: o perfil da educação superior apresentado pelos resultados do Enade 2005 e 2006. Revista Diálogo Educacional. Paraná, Brasil, v. 11, n. 32, pp. 187-206.

Soares, J. F., 2004. O efeito da escola no desempenho cognitivo de seus alunos. Revista Iberoamericana sobre Calidad, Eficacia y Cambio en Educación (REICE). Michigan, Estados Unidos, pp. 87-90.

Vista, N. P. B.; Figueiró, M. F.; Chicon, P. M. M., 2017. Técnicas de mineração de dados aplicadas aos microdados do ENADE para avaliar o desempenho dos acadêmicos do curso de Ciencia da Computação no Rio Grande do Sul utilizando o software R. I Seminário de Pesquisa Científica e Tecnológica. Rio Grande do Sul, Brasil. 